\title{
MÉTODOS TERAPÊUTICOS UTILIZADOS EM SUJEITOS COM DEFICIÊNCIA SENSÓRIO MOTORA APÓS DISFUNÇÃO VASCULAR ENCEFÁLICA: REVISÃO DE LITERATURA
}

\section{THERAPEUTIC METHODS USED IN SUBJECTS WITH MOTOR DISABILITIES AFTER CEREBRAL VASCULAR DYSFUNCTION: LITERATURE REVIEW}

\author{
Izabela dos Santos Mendes ${ }^{1}$ \\ Mariana Cesar Ribeiro dos Reis ${ }^{1}$ \\ Djenifer Queiroz de Souza ${ }^{2}$ \\ Ana Carolina Lacerda Borges ${ }^{3}$ \\ Sérgio Takeshi Tatsukawa de Freitas ${ }^{4}$ \\ Fernanda Pupio Silva Lima ${ }^{5}$ \\ Mário Oliveira Lima ${ }^{5}$
}

RESUMO: Atualmente vem crescendo novos métodos terapêuticos para aplicabilidade clínica em pacientes com Disfunção Vascular Encefálica (DVE). Grande parte desses pacientes apresentam hemiplegia espástica, com quadro de hipertonia espástica, sendo a DVE uma lesão das vias piramidais (córtex frontal) que provoca uma hiperexcitabilidade das vias neuronais, gerando hipertonia elástica, hiperreflexia, clônus, sinal de babinsk e alteração viscoelástica do músculo. Devido a esses sinais, os pacientes apresentam ausência da inibição recíproca que promove incapacidade sensório motora, gerando déficits funcionais permanentes. $A$ nova proposta para o tratamento de reabilitação é a terapia funcional para que possa estimular a funcionalidade mesmo com sequelas crônicas, pois esses exercícios estimulam a inibição recíproca, na qual ocorre a contração de um grupo muscular com inibição de seu antagonista. O objetivo deste estudo foi analisar a efetividade dos exercícios funcionais aplicados em indivíduos com hemiplegia espástica após DVE. Foi realizada uma revisão de literatura descritiva nas bases de dados LILACS, SciELO, MEDLINE, BIREME, do período de 1994 a 2011, com suas respectivas estratégias, referências de artigos de revistas especializadas e livros. De acordo com os resultados obtidos dos artigos pesquisados, acreditamos que os exercícios funcionais possam estimular a neuroplasticidade, melhorando suas atividades físicas e os aspectos psicossociais, porém, com sequela permanente. A terapia funcional envolve a contração de vários músculos durante a realização de cada exercício e isso faz com o indivíduo aprenda quais necessitam de contração durante suas atividades (aprendizagem motora), ou seja, gerando a inibição recíproca. Sugerimos, assim, que a terapia funcional possa ser útil no tratamento de hemiplegia espástica.

Palavras-chave: disfunção vascular encefálica; funcionalidade; cinesioterapia.

ABSTRACT: New therapeutic methods have been increasing for clinical application in patients with Cerebral Vascular Dysfunction (DVE). Most of these patients have spastic hemiplegia, with spastic hypertonia, and DVE in a pyramidal tract lesion (frontal cortex) that leads to hyperexcitability of neuronal pathways, generating elastic hypertonia, hyperreflexia, clonus, Babinski sign, and changes in the viscoelastic muscle. The signals of patients have no reciprocal inhibition which promotes sensory motor disabilities, generating permanent functional deficits. The new proposal for the rehabilitation treatment is functional therapy that can stimulate the

\footnotetext{
${ }^{1}$ Graduada em Fisioterapia - Universidade do Vale do Paraíba - Univap e Mestranda em Engenharia Biomédica - Univap. E-mails: izasmendes@hotmail.com.br; mah.crr@gmail.com.

${ }^{2}$ Graduada em Fisioterapia - Univap, Pós-graduanda em Fisioterapia em Neurologia - Instituto de Ensino e Pesquisa Hospital Israelita Albert Einstein e Mestranda em Engenharia Biomédica - Univap. E-mail: djeniferqs@gmail.com.

${ }^{3}$ Graduanda em Fisioterapia - Univap. Iniciação Cientifica PIBIC - Laboratório de Engenharia de Reabilitação Sensório Motora Univap. E-mail: carolborges.fisio@gmail.com.

${ }^{4}$ Doutorando em Engenharia Biomédica - Univap e professor da Univap. E-mail: takeshi@univap.br.

${ }^{5}$ Doutor(a) em Engenharia Biomédica e professor(a) da Univap. E-mails: fpupio@univap.br; mol@univap.br.
} 
functionality, even with chronic sequelae, because these exercises stimulate reciprocal inhibition, which is the contraction of a muscle group with inhibition of its antagonist. The objective of this study was to analyze the effectiveness of applied functional exercise in individuals with spastic hemiplegia after DVE. A descriptive review of the literature was performed in the LILACS, SCIELO, MEDLINE, and BIREME databases from 1994 to 2011 with their respective strategies, references to journal articles, and books. According to the results of the reviewed studies, we believe that functional exercises may stimulate neuroplasticity, improving physical activity and psychosocial aspects, but with permanent sequelae. The functional therapy involves the contraction of various muscles during the course of each exercise and this causes the patient to learn what contraction is needed during activities (motor learning), in other words, generating reciprocal inhibition. Therefore, we suggest that functional therapy may be useful in the treatment of spastic hemiplegia.

Keywords: cerebral vascular dysfunction; functionality; kinesiotherapy.

\section{INTRODUÇÃO}

A Disfunção Vascular Encefálica (DVE) é uma afecção vascular que promove alterações do fluxo sanguíneo, podendo ser isquêmico ou hemorrágico, atingindo o hemisfério encefálico, cujas características clínicas variam com 0 tamanho da localização da lesão e a quantidade de fluxo sanguíneo colateral (AFIFI; BERGMAN, 2008; BENSMAIL et al., 2010).

Segundo Kuriki et al. (2010), a lesão do córtex motor ou do trato corticoespinal pode resultar na perda de movimento do lado contralateral do corpo, caracterizada por uma hemiparesia ou hemiplegia, que é a consequência física mais comum de AVC, levando à incapacidade ou dificuldade em realizar diversas tarefas da vida diária (CARVALHO et al., 2007; KNUTSON; CHAE, 2010; STOKES, 2000).

O comprometimento mais evidente é a tendência em manter-se em uma posição de assimetria postural, com distribuição de peso menor sobre o hemicorpo parético. Essa assimetria e a dificuldade em transferir o peso para o lado afetado interferem na capacidade de manter o controle postural, impedindo a orientação e estabilidade para realizar movimentos com 0 tronco $\mathrm{e}$ membros (CESÁRIO; PENASSO; OLIVEIRA, 2006; GOMES et al., 2006;
TRINDADE et al., 2011).

O padrão de recuperação do controle motor relaciona as estruturas mais afetadas do sistema nervoso. O controle dos movimentos do corpo, no lado contralateral à lesão, atravessa estágios de recuperação das funções motoras e sensoriais que podem ser eficientes ou não. A espasticidade, característica da patologia, geralmente se desenvolve de forma lenta, após o período de hipotonia, com predileção pelos músculos flexores dos membros superiores e pelos músculos extensores dos membros inferiores, especialmente nos músculos antigravitatorios, permitindo padrões anormais de movimento (BOBATH, 2001; ESCARCEL; MULLER; RABUSKE, 2010; LIMA et al., 2008; LIMA, 2009; TEIXEIRA, 2008).

Segundo Bensmail et al. (2010), a espasticidade é descrita como desordem motora, caracterizada por um aumento velocidade-dependente, resultando em deficiência de inibição recíproca e o aumento da excitabilidade que leva à hiperreflexia, gerando consequências funcionais incapacitantes ao restringir a velocidade e a amplitude de movimentos voluntários. Os pacientes com sequelas de DVE apresentam redução do número de unidades motoras recrutadas, resultando em contração muscular ineficiente e limitação do desempenho 
funcional (FRANCIS et al., 2004; GAO; ZHANGI, 2008; GLANZ et al., 1996).

Existem vários métodos de intervenção fisioterapêutica ao paciente hemiplégico, no qual deve ser priorizada a funcionalidade. Dentre elas, a teoria neuroevolutiva que visa a facilitar os padrões normais de movimento e inibir os padrões espásticos e estereotipados. Estudos têm mostrado que o conceito Bobath é amplamente utilizado em reabilitação após DVE e outras condições neurológicas (GRAHAM et al., 2005; POLESE et al., 2008; SALMELA et al., 2000).

Brock et al. (2011) realizaram um estudo com 26 indivíduos, comparando os efeitos a curto prazo de duas abordagens de fisioterapia. $O$ estudo indicou que a intervenção baseada no conceito Bobath melhorou a velocidade de caminhada em pessoas com disfunção vascular encefálica.

Peres, Ruedell e Diamantes (2009) observaram o tônus e a força muscular, em diparéticos espásticos, após tratamento por Bobath, sendo evidenciado que o método proporcionou uma diminuição de tônus e aumento de força muscular, e consequente melhora nas atividades funcionais estáticas e dinâmicas.

Wang (1994) analisou a marcha de indivíduos com hemiplegia espástica, após realização da técnica de Facilitação Neuromuscular Proprioceptiva, observando, nos indivíouos em estágios agudos, melhora da velocidade e cadência da marcha imediatamente após uma sessão.

De acordo com Dobkin (2004) e Winstein et al. (2004) o treino motor deve ser o mais próximo possível das tarefas diárias realizadas pelo paciente, com benefícios motores em curto e longo prazo quando comparados a terapias não funcionais.

Deste modo a reabilitação por meio da Terapia Funcional pode ser uma abordagem complementar e que pode desenvolver uma melhora de longo prazo contribuindo assim com a qualidade de vida desses pacientes.

\section{OBJETIVOS}

O objetivo deste estudo foi avaliar por meio de revisão de literatura os efeitos de alguns exercícios funcionais aplicados em indivíduos com hemiplegia espástica após disfunção vascular encefálica.

\section{METODOLOGIA}

Trata-se de uma revisão sistemática e descritiva com objetivo de identificar, nas bibliografias disponíveis e atualizadas da língua espanhola, inglesa e portuguesa, o material de pesquisa.

O levantamento bibliográfico, propriamente dito, foi realizado por intermédio dos bancos de dados da SCIELO, LILACS, MEDLINE, BIREME e PUBMED, utilizando as palavras-chave, como Disfunção Vascular Encefálica, Espasticidade, Funcionalidade, Cinesioterapia, Kabat, Bobath. Foi, também, efetuado um levantamento manual de capítulos-texto de livros na biblioteca da Universidade do Vale do Paraíba (UNIVAP).

Os livros e artigos científicos foram adotados a partir do ano de 1994, progredindo até o ano 2011, e que estivessem relacionadas com o tema, bibliografias anteriores ao ano de 1994 foram excluídas desta revisão bibliográfica. 
Depois de uma análise criteriosa de 87 trabalhos pesquisados, foram selecionados aqueles que tinham as características evidenciadas das palavras-chave pesquisadas, resultando nos 40 trabalhos citados na referência, dos quais, 4 são livros texto.

\section{RESULTADOS}

De todos os 36 trabalhos utilizados, neste artigo, 16 abordavam a hemiplegia espástica decorrente de DVE, nos quais explicavam-se a fisiopatologia, o mecanismo da espasticidade e as principais alterações biomecânicas encontradas nesses pacientes. Nos outros 20 artigos, os autores relatavam sobre técnicas de reabilitação, sendo que, 12 artigos abordavam o Conceito Neuroevolutivo Bobath, 2 relatavam sobre Facilitação Neuromuscular Proprioceptiva e 6 artigos citavam, como base do tratamento, a funcionalidade, conforme demonstrado na Fig. 1.

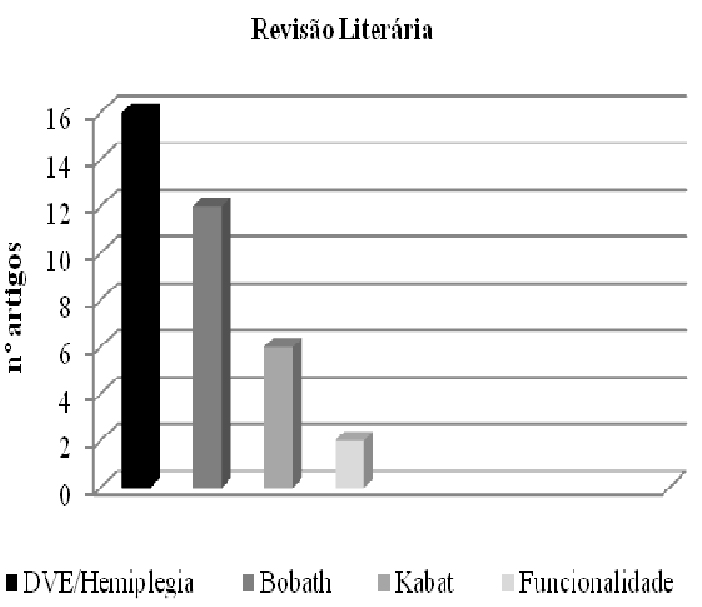

Fig. 1 - Distribuição porcentual da literatura pesquisada, conforme assuntos. 
Tabela 1 - Análise descritiva dos artigos pesquisados $(n=8)$ relacionados ao Conceito Neuroevolutivo Bobath

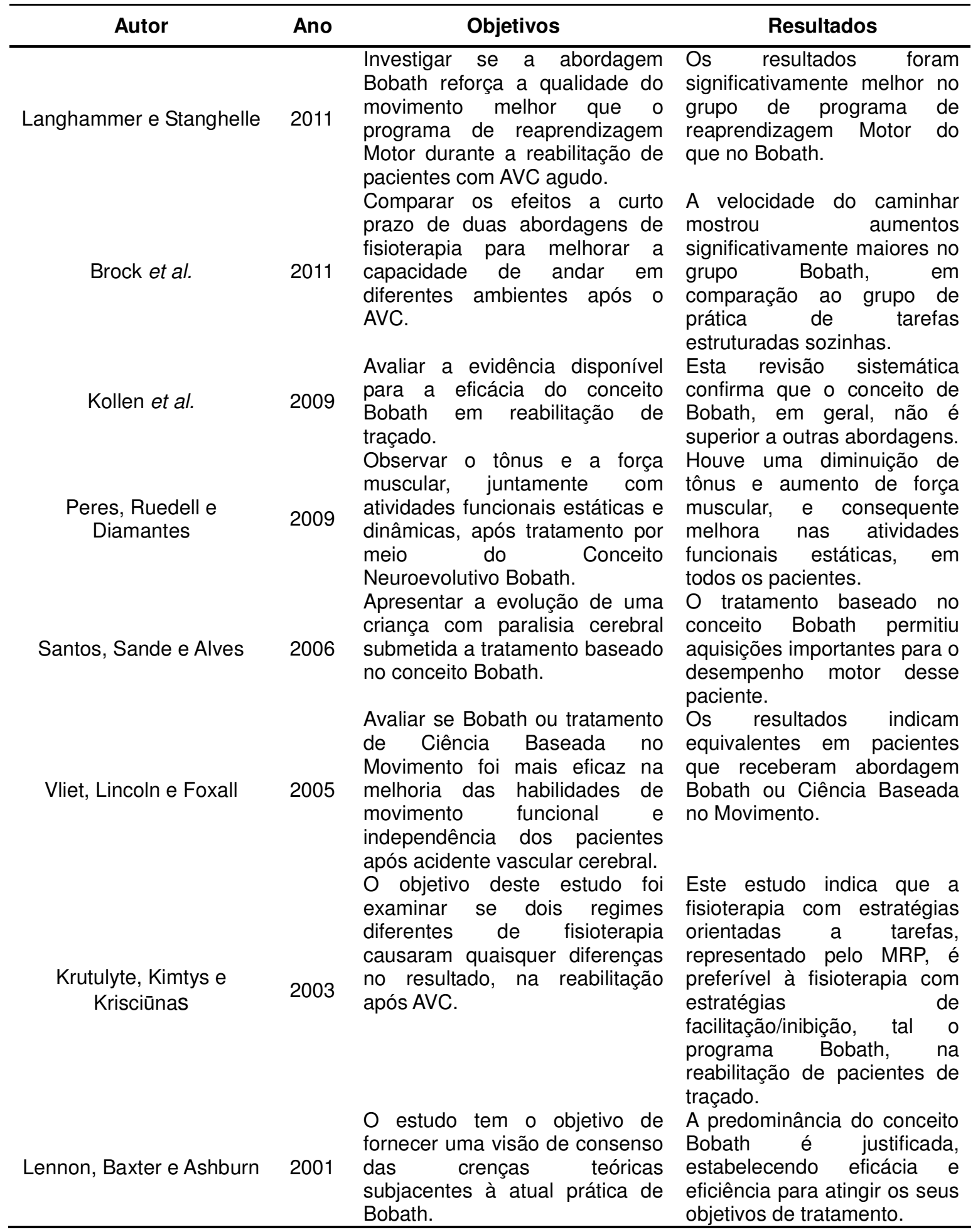


Tabela 2 - Análise descritiva dos artigos pesquisados $(n=5)$ relacionados ao Método Kabat e Funcionalidade

\begin{tabular}{|c|c|c|c|}
\hline Autor & Ano & Objetivo & Resultados \\
\hline Soares et al. & 2011 & $\begin{array}{l}\text { Analisar a utilização do método Kabat, no } \\
\text { tratamento fisioterapêutico, em crianças } \\
\text { com paralisia cerebral espástica. } \\
\text { Verificando, assim, a sua importância e os } \\
\text { seus benefícios. }\end{array}$ & $\begin{array}{l}\text { Foi comprovado que o } \\
\text { método Kabat baseia-se em } \\
\text { exercícios, utilizando vários } \\
\text { mecanismos facilitadores, } \\
\text { sendo benéficos ao paciente. }\end{array}$ \\
\hline Brianeze et al. & 2009 & $\begin{array}{l}\text { Verificar o efeito de um programa de } \\
\text { fisioterapia funcional para crianças com } \\
\text { PC, associado a orientações aos pais; e } \\
\text { verificar a correlação entre as habilidades } \\
\text { funcionais. }\end{array}$ & $\begin{array}{l}\text { A análise dos resultados } \\
\text { mostrou que as crianças } \\
\text { obtiveram } \\
\text { significativamente maiores } \\
\text { em comparação a avaliações } \\
\text { anteriores. }\end{array}$ \\
\hline Winstein et al. & 2004 & $\begin{array}{l}\text { Avaliar os efeitos imediatos e de longo } \\
\text { prazo com duas abordagens de } \\
\text { reabilitação da extremidade superior. }\end{array}$ & $\begin{array}{l}\text { O grupo tarefa funcional foi } \\
\text { mais benéfica a longo prazo, } \\
\text { em comparação ao grupo } \\
\text { resistência-força. }\end{array}$ \\
\hline $\begin{array}{l}\text { Magri, Silva e } \\
\text { Nielsen }\end{array}$ & 2003 & $\begin{array}{l}\text { Estudar a importância da integridade da } \\
\text { inervação recíproca na recuperação da } \\
\text { função motora do paciente hemiplégico por } \\
\text { AVC. }\end{array}$ & $\begin{array}{l}\text { Observou-se um resultado } \\
\text { satisfatório na diminuição da } \\
\text { espasticidade. }\end{array}$ \\
\hline Wang & 1994 & $\begin{array}{l}\text { Efeitos da FNM aplicada sobre a região } \\
\text { pélvica, de pacientes com marcha } \\
\text { hemiplegica de curta e longa duração. }\end{array}$ & $\begin{array}{l}\text { Os resultados foram } \\
\text { beneficos para os dois } \\
\text { grupos. }\end{array}$ \\
\hline
\end{tabular}

\section{DISCUSSÃO}

Nos achados do estudo, grande parte dos pacientes com DVE evolui com incapacidades e prejuízos sensório-motores, tendo como consequência um impacto significante em seu nível de independência funcional (OTTOBONI; FONTES; FUKUJIMA, 2002).

Atualmente, existem vários métodos que podem ser utilizados pelos fisioterapeutas para 0 tratamento de pacientes com hemiplegia espástica, os quais devem ser empregados de acordo com o quadro clínico apresentado pelo paciente, visando à funcionalidade, sendo os mais citados, na literatura, o método Neuroevolutivo Bobath e o Kabat (Facilitação Neuromuscular Proprioceptiva), apresentados na Tabelas 1 e 2 (ROTTA, 2002).

O Conceito Neuroevolutivo Bobath (representado na Tabela 1) é uma técnica de reabilitação neuromuscular, que avalia e trata pacientes com disfunção neural, com o objetivo de normalizar o tônus e facilitar o movimento, trabalhando os reflexos e reações. Esse método envolve um trabalho de reorganização sensoriomotora para reabilitação do paciente, respeitando, assim, sua capacidade motora (DIAS, 2007; SANTOS; SANDE; ALVES, 2006).

Langhammer e Stanghelle (2011) realizaram um estudo, a fim de investigar se a abordagem Bobath reforça a qualidade do movimento em comparação ao programa de reaprendizado motor, e foi observado que 0 grupo Bobath não obteve resultados significantes. Assim como Kollen et al. (2009), que, também, não observaram diferenças quando compararam o método a outra técnica de reabilitação.

Em contrapartida, Brock et al. (2011) 
compararam os efeitos a curto prazo de duas abordagens para melhorar a marcha hemiplégica em diferentes ambientes, resultando melhor velocidade ao caminhar, o grupo Bobath.

A facilitação neuromuscular proprioceptiva (FNM), representada na Tabela 2, é incorporada em padrões funcionais de movimento, baseados em movimentos normais, que são em espirais e diagonais. Esse método baseia-se em exercicios terapêuticos que utilizam diversos mecanismos facilitadores, promovendo meIhora da contração muscular, coordenação motora, equilíbrio e relaxamento muscular (SOARES et al., 2011).

Wang (1994) estudou sobre os efeitos da FNM aplicada sobre a região pélvica de pacientes que apresentavam marcha hemiplégica, encontrando resultados benéficos em sua pesquisa.

Portanto, todos esses métodos podem promover a inibição da espasticidade e a facilitação dos movimentos mais normais, levando ao reaprendizado do movimento seletivo, valendo-se do mecanismo de inibição recíproca (BRIANEZE et al., 2009).

$\mathrm{Na}$ inervação e inibição recíproca, as fibras la utilizam as vias colaterais, estabelecendo sinapses facilitatórias, ao mesmo tempo em que realizam, com a musculatura antagonista, sinapse inibitória, a fim de promover relaxamento e possibilitar uma contração adequada da musculatura agonista (EDWARDS, 1999; MORITA et al., 2006).

De acordo com os resultados obtidos dos artigos pesquisados, a inibição recíproca tem resultados satisfatórios na diminuição da espasticidade em pacientes hemiplégicos após a reaprendizagem (MAGRI; SILVA; NIELSEN, 2003).
A Terapia Funcional parte do mesmo princípio observado nas terapias Bobath e Kabath, as quais se fundamentam na funcionalidade do exercício. Desse modo, estes são executados a partir de posições adotadas nas atividades de vida diária, envolvendo a contração de vários grupos musculares ao mesmo tempo, promovendo a contração da musculatura agonista com o alongamento dos grupos antagonistas (COHEN, 2001; WINSTEIN et al., 2004).

Acredita-se que os exercícios funcionais possam estimular a neuroplasticidade nos indivíduos espásticos, melhorando as atividades físicas e os aspectos psicossociais, porém, com sequela permanente. A terapia funcional envolve a contração de vários músculos durante a realização de cada exercício e isso faz com o indivíduo aprenda quais grupos musculares necessitam de contração durante suas atividades (aprendizagem motora), ou seja, gerando a inibição recíproca (MORIELLO, FINCH; MAYO, 2011; TORRIANI et al., 2007).

Por intermédio dos estudos realizados sugere-se que a terapia funcional pode auxiliar na inibição da espasticidade, facilitando o controle motor e, consequentemente, as atividades de vida diária (BRIANEZE et al., 2009; COHEN, 2001).

\section{CONCLUSÃO}

Por meio dos artigos analisados, concluímos que a todas as técnicas são importantes no tratamento de pacientes com hemiplegia espástica decorrente de DVE. Porém sugerimos a Terapia Funcional como alternativa de escolha nos procedimentos que visam à funcionalidade em pacientes com sequelas crônicas. 


\section{REFERÊNCIAS}

AFIFI, A. K.; BERGMAN, R. A. Neuroanatomia Funcional: texto e atlas. São Paulo: Roca, 2007.

BENSMAIL, D. et al. Botulinum Toxin to Treat Upper-Limb Spasticity in Hemiparetic Patients: Analysis of Function and Kinematics of Reaching Movements. Neurorehabilitation and Neural Repair, v. 24, n. 3, p. 273-281, jan. 2010.

BOBATH, B. Hemiplegia em Adultos: Avaliação e Tratamento. 3. ed. São Paulo: Manole, 2001.

BRIANEZE, A. C. G. S. et al. E. Efeito de um programa de fisioterapia funcional em crianças com Paralisia Cerebral associado a orientações aos cuidadores: Estudo preliminar. Fisioter. pesq., v. 16, n. 1, p. 4045, jan/mar. 2009.

BROCK, K. et al. Does Pysiotherapy based on the Bobath concept, in conjunction with a task practice, achieve greater improvement in walking ability in people with stroke compared to physiotherapy focused on structured task practice alone? A pilot randomized controlled Trial. Clin Rehabil, v. 25, n. 10, p. 903-12, oct. 2011.

CARVALHO, A. C. et al. A. Projeto Hemiplegia - Um modelo de fisioterapia em grupo para hemiplégicos crônicos. Arq. Ciênc. Saúde, v. 14, n. 3, p. 161-168, jul/set. 2007.

CESÁRIO, C. M. M.; PENASSO, P.; OLIVEIRA, A. P. R. Impacto da disfunção motora na qualidade de vida em pacientes com Acidente Vascular Encefálico. Rev. Neurociências, v. 14, n. 1, p. 006-009, 2006.

COHEN, H. Neurociência para fisioterapeutas. São Paulo: Manole, 2001.
DIAS, A. A. S. Revisão Bibliográfica sobre o método Bobath - À luz da fisioterapia na Encefalopatia Crônica da Infancia Tipo Diplegia Espástica de 0 a 3 anos. 2007. Monografia de Conclusão de Curso de Fisioterapia - Universidade Veiga de Almeida, Rio de Janeiro.

DOBKIN, B. H. Strategies for Stroke Rehabilitation. Lancet Neurol., v. 3, n.9, p. 528-536, Set. 2004.

EDWARDS, S. Fisioterapia Neurológica: Uma abordagem centrada na resolução de problemas. São Paulo: Atheneu; 1999.

ESCARCEL, B. W.; MULLER, M. R.; RABUSKE, M. Análise do Controle Postural de pacientes com AVC Isquêmico próximo à alta hospitalar. Rev. neurociênc., v. 18, n. 4, p. 498-504, 2010.

FRANCIS, H. P. et al. Does reducing spasticity translate into functional benefit? An exploratory meta-analysis. J. Neurol. Neurosurg. Psychiatry, v. 75, p. 1547-1551, Jan. 2004.

GAO, F.; ZHANGI, L. Q. Altered contractile properties of the gastrocnemius muscle poststroke. J. appl. physiol., v. 15, n. 105, p. 1802-1808, Dez. 2008.

GLANZ, M. et al. Functional electrostimulation in post-stroke rehabilitation: A meta-analysis of the randomized controlled trials. Arch. phys. med. rehabil., v. 77, p. 549-553, Jun. 1996.

GOMES, B. M. et al. O Efeito da Técnica de Reeducação Postural Global em um paciente com Hemiparesia Após Acidente Vascular Encefálico. Acta Fisiatria, v. 13, n. 2, p. 103-108, jul. 2006.

GRAHAM, J. V. et al. The Bobath concept in contemporary clinical practice. Top. stroke rehabil., v. 16, n. 1, p. 57-68, Jan/Feb. 2005. 
KNUTSON, J. S.; CHAE, J. A Novel Neuromuscular Electrical Stimulation Treatment for Recovery of Ankle Dorsiflexion in Chronic Hemiplegia: A Case Series Pilot Study. Phys. med. rehabil., v. 89, n. 8, p. 672-682, Aug. 2010.

KOLLEN, B. J. et al. The effectiveness of the Bobath concept in stroke rehabilitation: what is the evidence? Stroke, v. 40, n. 4, p. 89-97, Apr. 2009.

KRUTULYTE, G.; KIMTYS, A.; KRISCIŪNAS A. The effectiveness of physical therapy methods (Bobath and motor relearning program) in rehabilitation of stroke patients. Medicina (Kaunas).. v. 39, n. 9, p. 889-895, 2003.

KURIKI, H. U. et al. The surface electromyography analysis of the non-plegic upper limb of hemiplegic subjects. Arq Neuropsiquiat., v. 68 , n. 4, p. 562-566, an. 2010.

LANGHAMMER, B.; STANGHELLE, J. K. Can Physiotherapy after Stroke Based on the Bobath Concept Result in Improved Quality of Movement Compared to the Motor Relearning Programme. Physiother. res. int., v. 16, p. 69-80, Jun. 2011.

LENNON, S.; BAXTER, D.; ASHBURN, A. Physiotherapy based on the Bobath concept in stroke rehabilitation: a survey within the UK. Disabil rehabil., v. 23, n. 6, p. 254-262, Apr. 2001.

LIMA, F. P. S. Eficácia de diferentes equipamentos biomédicos de reabilitação em pacientes com lesões no Sistema Nervoso Central. 2009. Tese (Doutorado em Engenharia Biomédica) Instituto de Pesquisa e Desenvolvimento, Universidade do Vale do Paraíba, São José dos Campos, 2009.

LIMA, M. O. et al. Efecto de La Estimulación
Eléctrica Neuromuscular y de los Ejercicios Isotônicos em los Músculos Flexores y Extensores de la Rodilla en Pacientes Hemipléjicos. Rev. neurol., v. 46, n. 3, p.135138, 2008.

MAGRI, M.; SILVA, N. S. S.; NIELSEN, M. B. P. Inervação recíproca na recuperação da função motora de paciente hemiplégico por Acidente Vascular Cerebral. Fisioter. Bras., v. 4, n. 3, p. 223-226, mai/jun. 2003.

MORIELLO, C.; FINCH, L.; MAYO, N. Relationship between muscle strength and functional walking capacity among people with stroke. J. Rehabil. Res. Dev., v. 48, n. 3, p. 267-276, nov. 2011.

MORITA, $\mathrm{H}$. et al. Lack of modulation of $\mathrm{lb}$ inhibition during antagonist contraction in spasticity. Neurology, v. 67, p. 52-56, Jul. 2006.

OTTOBONI, C.; FONTES, S. V.; FUKUJIMA, M. M. Estudo comparativo entre a Marcha Normal e a de Pacientes Hemiparéticos por Acidente Vascular Encefálico: Aspectos Biomecânicos. Rev. neurociências, v. 10, n, 1, p. 10-16, 2002.

PERES, L. W.; RUEDELL, A. M.; DIAMANTES, C. Influência do Conceito Neuroevolutivo Bobath no Tônus e Força muscular e Atividades Funcionais Estáticas e Dinâmicas em Pacientes Diparéticos espásticos após Paralisia Cerebral. Rev. Cent. Ciênc. Saúde (St. Maria), v. 35, n. 1, p. 28-33, 2009.

POLESE, J. C. et al. Avaliação da funcionalidade de indivíduos acometidos por Acidente Vascular Encefálico. Rev. neurociênc., v. 16, n. 3, p. 175-178, ago. 2008.

ROTTA, N. T. Paralisia Cerebral - Novas Perspectivas Terapêuticas. Jornal de pediatria, Rio de Janeiro, v. 78, 2002. In: 
SOARES, T. B. et al. A utilização do método Kabat (FNP) no tratamento fisioterapêutico em crianças com paralisia cerebral espástica: Revisão bibliográfica. Revista FisioBrasil, v. 14, n. 101, mar. 2011. Disponível em $<$ http://www.youblisher.com/p/155739-

Revista-FisioBrasil-Ed-101>. Acesso em: 21 maio 2012.

SANTOS, M. M.; SANDE, L. A. P.; ALVES, C. R. J.; Habilidades motoras em portador de Paralisia Cerebral submetido a tratamento baseado no Conceito Bobath: estudo de caso. Temas desenvolv., v. 15, n. 87/88, p. 38-41, jul/out. 2006.

SOARES, T. B. et al. A utilização do método Kabat (FNP) no tratamento fisioterapêutico em crianças com paralisia cerebral espástica: Revisão bibliográfica. Revista FisioBrasil, v. 14, n. 101, mar. 2011. Disponível em: set. 2005 .

$<$ http://www.youblisher.com/p/155739-

Revista-FisioBrasil-Ed-101>. Acesso em: 21 maio 2012.

STOKES, M. Neurologia para Fisioterapeutas. São Paulo: Premier, 2000.

TEIXEIRA, I. N. D. O. O Envelhecimento cortical e a reorganização neural após o Acidente Vascular Encefálico (AVE): Implicações para a reabilitação. Ciênc. saúde coletiva., v. 13 , n. 2, p. 2171-2178, dez. 2008.

SALMELA, T. L. F. et al. Fortalecimento muscular e condicionamento físico em hemiplégicos. Acta Fisiátrica, v. 7, n. 3, p. 108-118, 2000.

TORRIANI, C. et al. Relação entre independência e o nível de disfunção motora e funcional em pacientes hemiparéticos. Rev. Neurocienc., v. 15, n. 1, p. 33-38, jan./mar., 2007.

TRINDADE, A. P. N. T. et al. Influência da simetria e transferência de peso nos aspectos motores após Acidente Vascular Cerebral. Rev. Neurociências, 2011.

VLIET, P. M. V.; LINCOLN, N. B.; FOXALL, A. J. Comparison of Bobath based and movement science based treatment for stroke: a randomised controlled trial. Neurol. Neurosurg. Psychiatry, v. 76, p. 503-508, WANG, R. Y. Effect of Proprioceptive Neuromuscular Facilitation on the gait of patients with hemiplegia of long and short duration. Phys. ther., v. 74 , n. 12 , p. $1108-$ 1115, 1994.

WINSTEIN, C. et al. A randomized controlled comparison of upper-extremity rehabilitation strategies in acute stroke: a pilot study of immediate and long-term outcomes. Arch. phys. med. rehabil., v. 85, p. 620-628, 2004. 